\title{
Thermoregulatory responses in wheelchair tennis players: a pilot study
}

\author{
MT Veltmeijer ${ }^{1}$, B Pluim ${ }^{2}$, DH Thijssen ${ }^{1,3}$, MT Hopman $^{1}$ and TM Eijsvogels ${ }^{1,4}$
}

Study design: This was an observational study.

Objectives: Spinal cord-injured (SCl) individuals are thermoregulatory compromised because of an inability to vasodilate and sweat below the injury, increasing the risk, proportional to the injury level, for marked core body temperature (CBT) rises. We compared thermoregulatory responses between wheelchair tennis players with and without a $\mathrm{SCl}$.

Setting: British Open 2013, Nottingham, UK.

Methods: A total of 8 ( $3 \mathrm{SCl}$ and 5 non-SCl) wheelchair tennis players played a 45 -min match while we continuously measured CBT, 8-point skin temperature (Mean-Tsk) and exercise intensity (metabolic equivalent units (METs)). Thermal sensation and perceived exertion were measured before and after each set. Video-assisted logging of each serve, stroke and point duration was used to determine match intensity. No statistics were performed for CBT because of small sample sizes.

Results: Wet Bulb Globe Temperature varied between 18 and $20^{\circ} \mathrm{C}$. CBT increased stronger in the $\mathrm{SCl}$ players $\left(+0.6 \pm 0.1^{\circ} \mathrm{C}\right.$; $n=2)$ compared with the non-SCl players $\left(+0.3 \pm 0.1^{\circ} \mathrm{C} ; n=4\right)$, whereas Mean-Tsk was similar between groups $(P=0.29)$. No Tsk differences were observed above $(>\mathrm{T} 6)$ or below $(\leqslant T 6)$ the lesion level. Thermal sensation, perceived exertion, exercise and match intensity were similar between groups (all $P>0.05$ ).

Conclusions: In this small, descriptive study, CBT increased slightly more in the $\mathrm{SCl}$ wheelchair tennis players compared with non$\mathrm{SCl}$ players during a 45-min match in moderate environmental conditions. Further research to investigate whether $\mathrm{SCl}$ players are more prone to heat illness is warranted.

Spinal Cord (2014) 52, 373-377; doi:10.1038/sc.2014.27; published online 25 March 2014

Keywords: thermoregulation; core body temperature; hyperthermia; spinal cord injury; paraplegia

\section{INTRODUCTION}

The major tournaments in tennis, such as the Australian and US Open, are often played under highly demanding conditions, where ambient temperatures of $>35^{\circ} \mathrm{C}$ have been reported. An elevated core body temperature (CBT) is known to reduce exercise performance or may even pose a threat to any athlete's well-being by progressing to heat illness, and in extreme cases multiorgan failure or even death. ${ }^{1-3}$ Mediated by local and systemic (that is, neural) pathways, the body's principal thermoregulatory mechanisms in response to an elevated CBT during exercise are cutaneous vasodilatation and active sweat secretion.., 5

In spinal cord-injured (SCI) individuals, the neural transmission of thermoregulatory signals is interrupted, leading to an inability to vasodilate and sweat below the level of the lesion. ${ }^{6}$ Higher lesion levels therefore produce a larger body surface area that cannot actively regulate body temperature, resulting in a greater risk for heat-related problems. In laboratory settings, upper body exercise of similar intensity in SCI individuals and able-bodied controls was shown to result in a substantially higher increase in CBT in SCI subjects. ${ }^{7-9}$ However, no previous study has assessed the thermal strain in wheelchair tennis players with and without a SCI during a match. This is of special importance as the major tournaments for elite wheelchair tennis players are frequently played under hot and humid ambient conditions, for instance in view of the 2016 Paralympics in Rio de Janeiro.

The aim of this pilot study was to compare the thermoregulatory responses in wheelchair tennis players with and without a SCI during an outdoor tennis match. We hypothesized that SCI players would exhibit a larger CBT rise during the match compared with the non-SCI players.

\section{MATERIALS AND METHODS}

Subjects

Eight elite, international-level wheelchair tennis players (three SCI and five non-SCI) were included in the study (Table 1). All measurements were performed on-site during a single day at the British Open 2013 in Nottingham, United Kingdom. Because of the use of the telemetry system for measuring CBT, subjects with a history of (inflammatory) bowel disease or any abdominal surgery were excluded from this specific measurement. This study was approved by the ethics committee of the Radboud University Medical Centre, and all subjects provided a written informed consent before participation. We certify that all applicable institutional and governmental regulations concerning the ethical use of human volunteers were followed during the course of this research.

\footnotetext{
${ }^{1}$ Department of Physiology, Radboud University Medical Centre, Nijmegen, The Netherlands; ${ }^{2}$ Royal Netherlands Lawn Tennis Association, Amersfoort, The Netherlands; ${ }^{3}$ Research Institute for Sport and Exercise Science, Liverpool John Moores University, Liverpool, UK and ${ }^{4}$ Henry Low Heart Centre, Department of Cardiology, Hartford Hospital, Hartford, CT, USA

Correspondence: Dr TM Eijsvogels, Department of Physiology (392), Radboud University Medical Centre, PO Box 9101, 6500 HB Nijmegen, The Netherlands. E-mail: Thijs.Eijsvogels@Radboudumc.nl

Received 25 December 2013; revised 3 February 2014; accepted 11 February 2014; published online 25 March 2014
} 
Table 1 Individual subjects characteristics

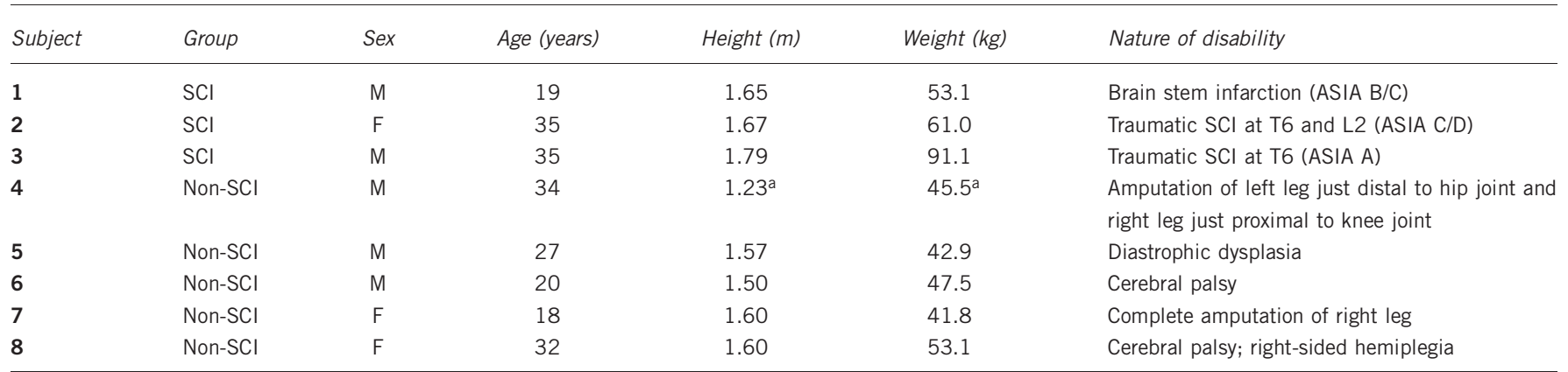

Abbreviations: ASIA, American Spinal Injury Association; F, female, M, male; Non-SCI, non-spinal cord injured; SCl, spinal cord injured a Measured without prosthesis.

\section{Experimental procedures and materials}

All measurements were performed at outside courts. Subjects were instructed to warm-up for a fixed 5-min period and subsequently play a simulated 45-min singles match against a similarly ranked opponent who also participated in the study. Measurements were started before the warming-up and continued throughout the match.

Core body temperature. Participants ingested an individually calibrated telemetric temperature pill that measured (intestinal) CBT and transmitted the temperature via radio waves to an external recorder (CorTemp; HQ, Palmetto, FL, USA) that was attached underneath each participant's wheelchair. This measuring system has been demonstrated to safely and reliably measure CBT during exercise and rest. ${ }^{10,11}$ The wireless signal was recorded every $20 \mathrm{~s}$ and was post hoc averaged for each min.

Skin temperature. We measured skin temperature (Tsk) every 30 s throughout the match by attaching wireless data loggers directly onto the skin (iButton; Maxim Integrated, San Jose, CA, USA) using Tegaderm transparent film dressing (Tegaderm Film 1624, Neuss, Germany). Use of these data loggers to measure Tsk has been validated and described in previous studies. ${ }^{12}$ Tsk was measured at a resolution of $0.0625^{\circ} \mathrm{C}$ by attaching the data loggers on the forehead, right scapula, upper left thorax, right upper arm, left forearm, left hand, ventral side right upper leg and left calf. Mean-Tsk was calculated according to ISO 9886 and averaged for each min. ${ }^{13}$ Tsk was further divided into an upper region above the lesion level (>T6) and a lower region below the lesion level ( $\leqslant \mathrm{T} 6$; that is, right upper leg and left calf) to identify any differences in Tsk relating to the SCI. The level of T6 was chosen as all our subjects had their SCI distal to this level.

Thermal sensation, perceived exertion and exercise intensity. Thermal sensation (7-point scale: -3 cold; +3 hot) and rating of perceived exertion (15-grade BORG scale) were assessed after each completed set. ${ }^{14,15}$ Exercise intensity was determined using an accelerometer (SenseWear Pro3; Bodymedia, Pittsburg, PA, USA) that expressed exercise intensity in metabolic equivalent units (METs) for every min played. The accelerometer was worn on the right upper arm throughout the match. Previous studies have demonstrated this method to validly and accurately measure energy expenditure including in wheelchair users. ${ }^{16}$

Video-assisted logging of match intensity. In addition to the accelerometry data, alternative descriptive parameters concerning the intensity of the matches were obtained by video recording all matches. A specifically developed tabletapp was used to manually tag the time point on which a point started (serve) and on which a point ended (ball was out, hit the net, hit the ground $\geqslant 3$ times or hit the ground behind the player). These data were used to extract the average rally and rest duration. Stroke frequency was determined by manually counting the number of times that a ball was hit during each rally divided by the rally duration. These parameters were defined and determined in accordance with previous literature. ${ }^{17}$
Environmental conditions. Wet Bulb Globe Temperature (WBGT) was measured using a portable weather station (Davis Instruments, Hayward, CA, USA) every $60 \mathrm{~min}$ throughout the day. This measurement allows for standardization of environmental conditions by accounting for outside temperature $\left(T_{D R Y-B U L B}\right)$, outside temperature with correction for humidity $\left(T_{\text {WET-BULB }}\right)$ and (solar) radiation $\left(T_{\mathrm{GLOBE}}\right)$. WBGT was calculated using the following formula: WBGT $=0.1 \quad\left(T_{\text {DRY-BULB }}\right)+0.7 \quad\left(T_{\text {WET-BULB }}\right)+0.2$ $\left(T_{\mathrm{GLOBE}}\right){ }^{3}$

\section{Statistics}

Statistical analyses were performed using IBM SPSS version 20.0 (IBM Corp., Armonk, NY, USA). Data were reported as mean \pm s.d. unless otherwise indicated. Because of the small sample size, no statistical analyses could be performed for the CBT data. Normality distribution was tested using the Shapiro-Wilk test. All normally distributed data were analysed using parametric tests ( $t$-test and two-way repeated measurements analysis of variance), and in case of non-Gaussian distribution nonparametric tests were applied (Mann-Whitney $U$-test). The level of significance was set at $P \leqslant 0.05$.

\section{RESULTS}

Two subjects (one SCI and 1 non-SCI) were excluded from CBT measurements because of prior abdominal surgery. All measurements were performed in moderate environmental conditions (WBGT 17.9$20.0^{\circ} \mathrm{C}, T_{\text {DRY-BUlB }} 21.2-24.8^{\circ} \mathrm{C}, T_{\mathrm{GLOBE}} 21.7-26.3^{\circ} \mathrm{C}$, relative humidity $51.8-61.4 \%$ ) with a complete cloud overcast.

The individual CBT for the SCI subjects and mean CBT for the non-SCI subjects are reported in Figure 1, and showed a slightly larger CBT increase in the SCI subjects $\left(+0.6 \pm 0.1^{\circ} \mathrm{C} ; n=2\right)$ compared with the non-SCI subjects $\left(+0.3 \pm 0.1{ }^{\circ} \mathrm{C} ; n=4\right)$.

Before the match, we found no significant differences between groups in mean-Tsk $(P=0.58)$. A significant change in mean-Tsk was reported over time $(P<0.001$; Figure $2 \mathrm{a})$, whereas no differences were observed between SCI and non-SCI players $(P=0.29)$. Tsk in the upper region changed significantly over time $(P<0.001$; Figure $2 b)$, whereas no differences between both groups were reported $(P=0.66)$. No differences were reported in the lower region Tsk over time $(P=0.42)$ or between groups over time $(P=0.99)$.

Thermal sensation increased similarly from 'cold' to 'warm' in both groups (Table 2). Rating of perceived exertion was similar at baseline (SCI $9.3 \pm 2.9$, non-SCI $8.6 \pm 1.8 ; P=0.54$ ) and increased to similar levels at the end of the second set (SCI $13.7 \pm 1.2$, non-SCI $13.2 \pm 2.5$; $P=0.61$ ). Average exercise intensity levels (in METs) were similar throughout the match in both groups $(4.9 \pm 0.7$ versus $4.9 \pm 0.7$; $P=1.00)$. Match intensity parameters are reported in Table 2, and showed no differences between both groups. 


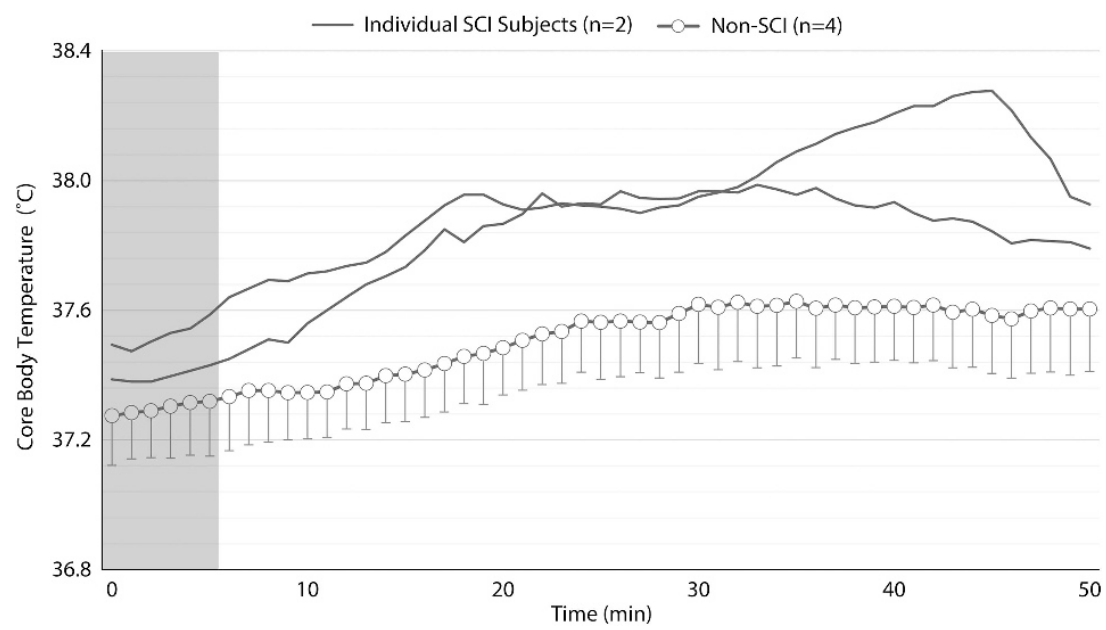

Figure 1 Core body temperature throughout the match in the two individual SCl subjects (solid lines) and the non-SCl group (open circles). The grey area represents the warming-up period. Data are presented as individual values (SCl subjects) and mean \pm s.e.m. (non-SCl subjects).
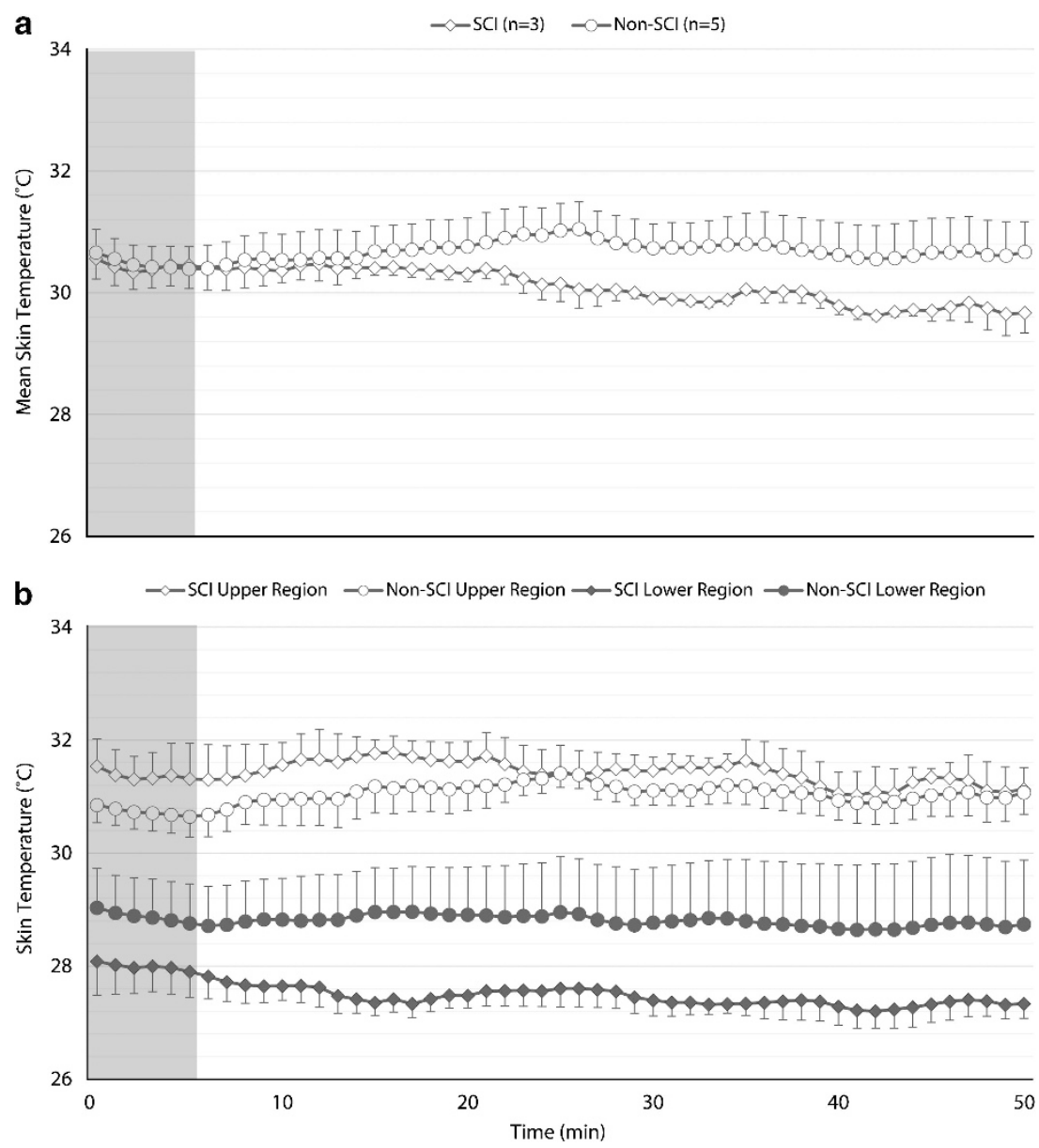

Figure 2 Mean skin temperature (a) throughout the match in the SCl group (open squares) and the non-SCl group (open circles). The grey area represents the warming-up period. Mean-Tsk changed significantly throughout the match in both groups over time (two-way repeated measurements analysis of variance (2W RM ANOVA): $P<0.001$ ), whereas no differences were observed between $\mathrm{SCl}$ and non-SCl players (time $\times$ group: $P=0.29$ ). Skin temperatures in the upper skin region above ( $>\mathrm{T} 6$ ) and in the lower skin region below ( $\leqslant \mathrm{T} 6)$ the level of the spinal cord injury are shown (b) for the $\mathrm{SCl}$ group (upper region open squares; lower region closed squares) and the non-SCl group (upper region open circles; lower region closed circles). A significant change was observed in both groups in the upper region, but no differences were observed between the $\mathrm{SCl}$ and non-SCl players. In the lower region, no differences were observed throughout the match $(P=0.42)$ or between both groups of players $(P=0.99)$. Data are presented as mean \pm s.e.m. 
Table 2 Match and exercise intensity parameters throughout the match, thermal sensation and rating of perceived exertion at baseline, after the first set and immediately after the match

\begin{tabular}{|c|c|c|c|}
\hline & $S C l(n=3)$ & Non-SCI $(\mathrm{n}=5)$ & P-value \\
\hline \multicolumn{4}{|l|}{ Match intensity } \\
\hline Duration of rallies (s) & $8.4 \pm 1.1$ & $9.8 \pm 2.1$ & 0.36 \\
\hline Rest time between points (s) & $21.7 \pm 2.8$ & $20.1 \pm 3.0$ & 0.36 \\
\hline Work/rest ratio & $1: 4.0 \pm 1.5$ & $1: 3.3 \pm 1.4$ & 0.36 \\
\hline Effective playing time ( $\%$ of total time) & $21.3 \pm 7.6$ & $25.2 \pm 7.5$ & 0.36 \\
\hline Strokes per rally $(n)$ & $3.7 \pm 0.8$ & $4.0 \pm 0.7$ & 0.76 \\
\hline Stroke frequency (str/min) & $26.1 \pm 2.2$ & $24.6 \pm 2.6$ & 0.36 \\
\hline Exercise intensity (METs) & $4.9 \pm 0.7$ & $4.9 \pm 0.7$ & 1.00 \\
\hline \multicolumn{4}{|l|}{ Thermal sensation } \\
\hline Baseline & $-1.0 \pm 0.0$ & $-0.8 \pm 1.3$ & 0.33 \\
\hline After first set & $1.7 \pm 1.2$ & $1.2 \pm 0.4$ & 0.56 \\
\hline After second set & $1.0 \pm 1.7$ & $1.6 \pm 0.5$ & 0.86 \\
\hline \multicolumn{4}{|l|}{ Rating of perceived exertion (BORG) } \\
\hline Baseline & $9.3 \pm 2.9$ & $8.6 \pm 1.8$ & 0.54 \\
\hline After first set & $12.3 \pm 2.3$ & $12.8 \pm 1.8$ & 0.76 \\
\hline After second set & $13.7 \pm 1.2$ & $13.2 \pm 2.5$ & 0.61 \\
\hline
\end{tabular}

Abbreviations: METs, metabolic equivalent units; Non-SCI, non-spinal cord injured; $\mathrm{SCl}$, spinal cord injured.

$P$-values refer to the Mann-Whitney U-test.

Data are presented as mean \pm s.d.

\section{DISCUSSION}

The present study was the first to describe changes in CBT during exercise in wheelchair tennis players with and without a SCI. Our results are indicative that wheelchair tennis causes a rise in CBT under moderate environmental conditions, and that this rise may be stronger in SCI players compared with non-SCI players in spite of similar Tsk responses. These findings may have clinical and practical importance for SCI players, particularly while playing tennis matches that are organized in hot and humid conditions as can be expected during most Grand Slam tournaments or the 2016 Paralympics in Rio de Janeiro.

CBT showed a larger rise in the SCI subjects compared with the non-SCI subjects. Although it is difficult to draw any firm conclusions based on these descriptive data, these results are in support of our hypothesis and are in agreement with findings in previous laboratorybased studies. ${ }^{7-9,18}$ In able-bodied subjects, exercise intensity is strongly correlated to CBT increases. ${ }^{3}$ The similar exercise intensity, match intensity and rating of perceived exertion between both groups suggest that these factors cannot explain the larger CBT rise in the SCI players.

Furthermore, it has been previously described that although paraplegic athletes show CBT responses similar to able-bodied athletes during exercise in moderate to hot conditions, the SCI athletes have a greater heat storage in the lower body that results in a reduced ability to lower their CBT during recovery periods. ${ }^{9}$ This observation is of particular importance in wheelchair tennis, as tennis is typically characterized by bouts of exercise (rallies) alternated by periods of relative rest (for example, ball retrieval and preparation phase for next serve). This means that although the non-SCI players may be able to cool down during the rest periods in between rallies, the SCI players will not be able to do so as effectively. Therefore, an attenuated heat loss capacity in the SCI players, resulting from a severely reduced body surface area for active thermoregulation and increased lower body heat storage, may be responsible for the observed CBT rise in our SCI subjects. As our measurements were performed under moderate conditions, differences between SCI and non-SCI wheelchair players may be exaggerated under hot conditions, placing SCI wheelchair players at potential risk for an impaired performance or developing heat illness during tournaments in the heat. Lastly, as the test matches in the present study were relatively short in duration compared with a typical wheelchair tennis match ( $\sim 75 \mathrm{~min}$ ), our results may have underestimated the CBT that would be attained toward the end of the matches.

Skin temperature responses were similar between SCI and non-SCI players. As Tsk is largely related to the ambient temperature, no group differences were expected. ${ }^{19}$ Furthermore, any differences between both groups in upper and lower body Tsk responses would have likely resulted from the inability to vasodilate or sweat below the lesion level in the SCI players. Previous studies demonstrated that lower body Tsk increases during prolonged (90-min) upper body exercise as a result of increased heat storage in that region. ${ }^{9,20}$ Although both heat load and exercise duration during the present study were limited, this may explain lack of pronounced Tsk responses in the SCI and non-SCI group.

Our study had some limitations. An obvious limitation of our study is the low number of subjects that makes it difficult to draw strong conclusions about the actual risk of heat stress that the SCI players are subjected to during a match. Further research with larger sample size should be performed to gain a better insight into the thermal stress that wheelchair players are exposed to and the associated risks for developing heat illness including collapse and heat stroke.

In conclusion, we provide some evidence for a larger increase in CBT in SCI wheelchair tennis players compared with non-SCI players while playing a 45-min tennis match in moderate ambient conditions. This finding suggests that SCI players may have an elevated risk of developing heat illness, particularly under hot ambient conditions. We believe that our findings warrant future studies to specifically examine the thermoregulatory responses in SCI wheelchair tennis players, and also highlight the development of countermeasures to prevent hyperthermia.

\section{DATA ARCHIVING}

There were no data to deposit.

\section{CONFLICT OF INTEREST}

The authors declare no conflict of interest.

\section{ACKNOWLEDGEMENTS}

We express our gratitude to the following individuals who assisted with all measurements: Bart Philips, Martijn Maessen and Jet Bouwens. We also thank Aldo Hoekstra and Elmy van Tok (Video Support KNLTB) for video recording and tagging all matches. We thank the International Tennis Federation and the Lawn Tennis Association for providing their endorsements to this study. This study was funded by the Royal Netherlands Lawn Tennis Association, Amersfoort, The Netherlands; NOC*NSF, Arnhem, The Netherlands; the International Tennis Federation, London, United Kingdom; and the Radboud University Nijmegen Medical Centre, Nijmegen, The Netherlands. Dr Eijsvogels received a Rubicon Grant (825.12.016). Dr Thijssen is financially supported by The Netherlands Heart Foundation (E. Dekker-stipend 2009/065).

1 Schlader ZJ, Raman A, Morton RH, Stannard SR, Mundel T. Exercise modality modulates body temperature regulation during exercise in uncompensable heat stress. Eur J Appl Physiol 2011; 111: 757-766.

2 Tucker R, Rauch L, Harley YX, Noakes TD. Impaired exercise performance in the heat is associated with an anticipatory reduction in skeletal muscle recruitment. Pflugers Arch 2004; 448: 422-430. 
3 Armstrong LE, Casa DJ, Millard-Stafford M, Moran DS, Pyne SW, Roberts WO. American College of Sports Medicine position stand. Exertional heat illness during training and competition. Med Sci Sports Exerc 2007; 39: 556-572.

4 Kellogg Jr DL. In vivo mechanisms of cutaneous vasodilation and vasoconstriction in humans during thermoregulatory challenges. J Appl Physiol 2006; 100: 1709-1718.

5 Johnson JM, Kellogg Jr DL. Thermoregulatory and thermal control in the human cutaneous circulation. Front Biosc 2010; 2: 825-853.

6 Sawka MN, Latzka WA, Pandolf KB. Temperature regulation during upper body exercise: able-bodied and spinal cord injured. Med Sci Sports Exerc 1989; 21 : S132-S140.

7 Petrofsky JS. Thermoregulatory stress during rest and exercise in heat in patients with a spinal cord injury. Eur J Appl Physiol Occup Physiol 1992; 64: 503-507.

8 Price MJ, Campbell IG. Effects of spinal cord lesion level upon thermoregulation during exercise in the heat. Med Sci Sports Exerc 2003; 35: 1100-1107.

9 Price MJ. Thermoregulation during exercise in individuals with spinal cord injuries. Sports Med 2006; 36: 863-879.

10 Byrne C, Lim CL. The ingestible telemetric body core temperature sensor: a review of validity and exercise applications. Br J Sports Med 2007; 41: 126-133.

11 Gant N, Atkinson G, Williams C. The validity and reliability of intestinal temperature during intermittent running. Med Sci Sports Exerc 2006; 38: 1926-1931.
12 van Marken LWD, Daanen HA, Wouters L, Fronczek R, Raymann RJ, Severens NM et al. Evaluation of wireless determination of skin temperature using iButtons. Physiol Behav 2006; 88: 489-497.

13 ISO. ISO 9886: Evaluation of Thermal Strain by Physiological Measurements. ISO: Geneva, 1992.

14 Borg GA. Psychophysical bases of perceived exertion. Med Sci Sports Exerc 1982; 14 : 377-381.

15 Gagge AP, Stolwijk JA, Hardy JD. Comfort and thermal sensations and associated physiological responses at various ambient temperatures. Environ Res 1967; 1: 1-20.

16 Hiremath SV, Ding D. Evaluation of activity monitors to estimate energy expenditure in manual wheelchair users. Conf Proc IEEE Eng Med Biol Soc 2009; 2009: 835-838.

17 Kovacs M. A comparison of work/rest intervals in men's professional tennis. Med Sci Tennis 2004; 9: 10-11.

18 Price MJ, Campbell IG. Thermoregulatory responses during prolonged upper-body exercise in cool and warm conditions. J Sports Sci 2002; 20: 519-527.

19 Schlader ZJ, Stannard SR, Mundel T. Human thermoregulatory behavior during rest and exercise - a prospective review. Physiol Behav 2010; 99: 269-275.

20 Price MJ, Campbell IG. Thermoregulatory responses of paraplegic and able-bodied athletes at rest and during prolonged upper body exercise and passive recovery. Eur $J$ Appl Physiol Occup Physiol 1997; 76: 552-560. 